NISTIR 7618

\title{
STEP-OAGIS Harmonization Joint Working Group
}

\author{
PDM Subgroup Interim Report
}

Xenia Fiorentini

Sudarsan Rachuri 
NISTIR 7618

\title{
STEP-OAGIS Harmonization Joint Working Group
}

\author{
PDM Subgroup Interim Report
}

\author{
Xenia Fiorentini \\ Sudarsan Rachuri \\ Manufacturing Systems Integration Division \\ Manufacturing Engineering Laboratory
}

Committee Members: Vijay Srinivasan, Alan Boyd, Annap Derebail, Charlie Stirk, David Connelly, Mike Rowell, Frank Heinrich, Steven Vettermann, Sudarsan Rachuri, Hyowon Suh, and Xenia Fiorentini

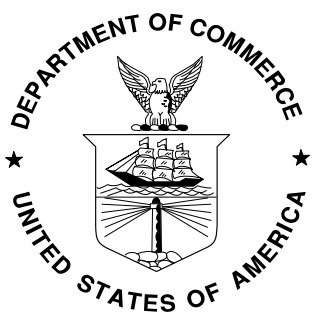

U.S. Department of Commerce

Gary Locke, Secretary

National Institute of Standards and Technology Patrick D. Gallagher, Deputy Director 


\section{Executive Summary}

In any manufacturing enterprise, there are two types of tools used to create and share product related data across its extended network. The engineering information of the product is created using what is commonly called an engineering authoring tool. Some examples of engineering authoring tools include Computer-Aided Design (CAD), Computer-Aided Engineering (CAE), Product Data Management (PDM), and Product Lifecycle Management (PLM). This information, encapsulated in engineering objects, mainly focuses on geometry information and some amount beyond geometry. The business information, encapsulated in business objects, is created using business authoring tools. Some examples of business authoring tools include Enterprise Resource Planning (ERP), Customer Relationship Management (CRM), and Supply Chain Management (SCM). Various solutions have been proposed to address interoperability issues within engineering tools and business tools. The prominent among them are standard-based, for example, ISO 10303 [1], informally known as STEP (STandard for Exchange of Product model data), for engineering tools.

The focus of this project is to address the bigger problem of interoperability between engineering tools and business tools. The short term focus of the project is to analyze the potential harmonization of the ISO STEP-based OMG (Object Management Group) standard, called PLM Services [2] (web services for engineering objects), and OAGIS (Open Application Group Integration Specification) standard [3] (XML exchange protocol for business objects). The output of this short-term project will identify common concepts between these two standards that need to be harmonized. A scenario to demonstrate this harmonization to enable sharing of information between engineering and business tools using PLM Services and OAGIS will be developed. The long-term goal of this project is to develop a method for comparing and harmonizing standards. This will help designers, engineers, and process modelers in selecting appropriate standards for interoperability. 


\section{Table of contents}

1 Introduction and Objectives................................................................................... 1

2 A brief introduction to OMG PLM Services and OAGIS ............................................ 2

2.1 OMG PLM Services in brief .......................................................................... 2

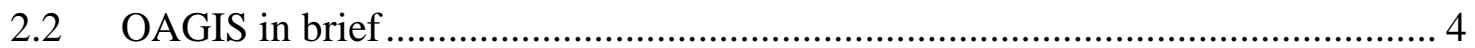

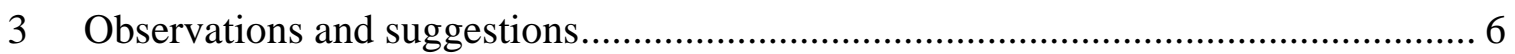

3.1 Common concepts between OMG PLM Services and OAGIS............................ 6

3.2 Modeling paradigm in OMG PLM Services and OAGIS .................................... 8

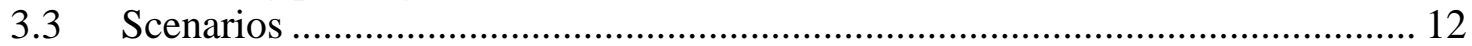

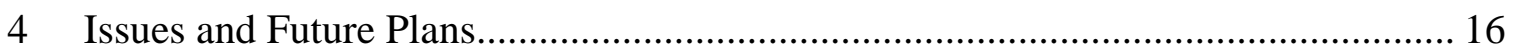

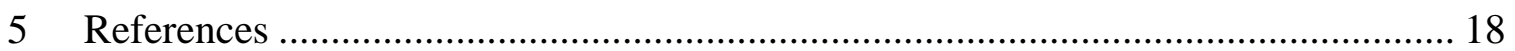

\section{List of figures}

Figure 1: UML version of the informational model in OMG PLM Services: part identification package .......................................................................................... 3

Figure 2: The corresponding XML version ............................................................ 4

Figure 3: Contents of a Business Object Document (BOD) [3] …………......................... 5

Figure 4: The GetItemMaster BOD ............................................................................... 6

Figure 5: Real world products and product models data .................................................... 9

Figure 6: Product model and real world product in OMG PLM Services ......................... 10

Figure 7: Physical_instance as a core concept for realizing the mapping .......................... 11

Figure 8: MapForce screenshot of the Physical_instance mapping................................... 12

Figure 9: ECM scenario in OAGIS......................................................................... 14

Figure 10: A scenario for this project ………………............................................... 15

\section{Table of tables}

Table 1: Examples of the product information represented in the ItemMaster noun .......... 7

Table 2: Examples of use cases in OMG PLM Services and scenarios in OAGIS .......... 13 


\section{Introduction and Objectives}

Global extended network enterprises participate in collaborative activities such as marketing, planning, development, manufacturing, and disposal of a product. Sharing and exchanging product and related information among these partners is essential to guarantee an efficient collaboration along the network. This information needs to be exchanged not only between different applications in the same domain, such as two different Computer-Aided Design (CAD) applications or between two different Enterprise Resource Planning (ERP) applications, but also between applications belonging to different domains, such as between CAD and ERP applications.

Many information standards have been created to integrate applications belonging to the same domain. Within the engineering domain, ISO-10303 (STEP) [1] represents by far the most accepted standard, widely implemented in CAD, CAE (Computer-Aided Engineering) and PDM (Product Data Management) systems. Many other standards within the engineering domain have been developed as spin-offs to STEP, for example OMG PLM Services [2]. Within the business domain, the standards landscape offers a major variety of standards, mostly proposed by open and nimble organizations such as OMG (Object Management Group) [4], OASIS (Organization for the Advancement of Structured Information Standards) [5], and OAGi (Open Applications Group Inc) [6].

While domain-specific standards have already been developed, the integration of standards belonging to different domains, although desired, remains unrealized. In [7], Srinivasan noted that "time is ripe for such integration because of the convergence of three important factors: maturity of the domain-specific standards, emergence of the SOA architecture for information sharing and availability of robust middleware to implement them.” This project exploits the opportunities offered by these three factors and addresses the need to integrate engineering and business information systems.

In particular, this project aims to facilitate the exchange of product data between PDM systems (engineering authoring tools) and ERP systems (business authoring tools) by harmonizing two standards widely adopted by each of them: PLM Services from OMG and OAGIS [3] from OAGi respectively.

The aim of OMG PLM Services is to make the engineering objects derived from STEP readily available over the web. Following the Model Driven Architecture (MDA) [8], OMG PLM Services provides both a platform-independent and a platform-specific view point. In the platform independent viewpoint, an informational model, directly derived from the STEP AP214 CC21 (conformance class 21) ARM (Application Reference Model) [9] model, describes the organization of the modeling concepts while a computational model, derived from PDTNet [10] and PDM Enablers [11], captures the functional aspects of the informational model. In the platform-specific viewpoint, the informational model of the platform-independent viewpoint is mapped into XML [12] while the computational model is mapped in WSDL(Web Services Description Language) [13].

The scope of OAGIS is that of structuring, in business documents, enterprise-wide business functions such as ERP, e-commerce, CRM (Customer Relationship Management), and finance. 
in business documents. The Business Object Documents (BODs) are business messages defined in XML and exchanged between business application systems. Each BOD includes an application area containing the information used to communicate the message, and a data area describing the content of the message itself. The content of the message is further organized into a noun identifying the business-specific data to be communicated, and a verb identifying the action being performed on the noun.

The goal of this project is to study and compare OMG PLM Services and OAGIS with the aim of harmonizing them. The specific objectives are:

- Understanding each standard by identifying its scope, organization, and modeling philosophy

- Designing one or more use cases (scenarios) to understand the integration issues

- Recognizing the concepts that intersect both the standards

- Identifying portions of the models that are part of the intersection

- Realizing the harmonization

To achieve these objectives, the working group composition brought together disparate competences: members of the organizations owning the standards, participants from organizations that are promoting the standards, and experts in product information modeling. The following people participated in the project: Vijay Srinivasan (then IBM), Alan Boyd (IBM), Annap Derebail (IBM), Charlie Stirk (CostVision), David Connelly (OAGi), Mike Rowell (Oracle/OAGi), Frank Heinrich (iBASEt/OAGi), Steven Vettermann (ProSTEP iViP), Sudarsan Rachuri (NIST), Hyowon Suh (NIST/ KAIST), and Xenia Fiorentini (NIST).

\section{A brief introduction to OMG PLM Services and OAGIS}

The team approached this project by considering and analyzing the two standards: OMG PLM Services version 2 and OAGIS version 9.2. Studying and reviewing these standards helped the team to understand the standards on their own, to analyze their differences, and to identify their intersection.

\subsection{OMG PLM Services in brief}

The scope of OMG PLM Services is to represent engineering objects and to integrate them with service technologies. To achieve this scope, the OMG PLM Services specification provides i) a model to structure the engineering information of a product and ii) a model to query and navigate the structure. These two models are called, respectively, the informational model and the computational model. The specification defines a Platform Specific Model (PSM) applicable to the web services implementation defined by a WSDL specification, with a SOAP (Simple Object Access Protocol) binding, and an XML schema specification. The services derived from the combination of the informational model and computational model allow creating, reading, updating, and deleting engineering objects.

OMG PLM Services is considered a spin-off of STEP since its informational model is directly derived from CC21 (Conformance Class 21) of STEP AP214 $3^{\text {rd }}$ edition [9]. This informational model represents the following: 
- part identification

- part structure

- document and file management

- shape definition and transformation

- product classification

- product properties

- authorization

- configuration management

- change and work management

- process planning

- multi-language support

To obtain the informational model, the AP214 [9] CC21 AIM (Application Interpreted Model) in EXPRESS [14] is mapped through EXPRESS-X [15] into the ARM (Application Reference Model). The obtained ARM model in EXPRESS is mapped through XMI (XML Metadata Interchange) [16] into UML (Unified Modeling Language) [17]. Finally, the informational model is represented in XML schema. Unfortunately, the different nature of the languages implies a loss of information during this final translation; the concepts network expressed in UML can be hard to translate into a concepts tree in XML.

We report here an example of the UML and XML versions of the informational model describing the part identification (Figure 1 and Figure 2).

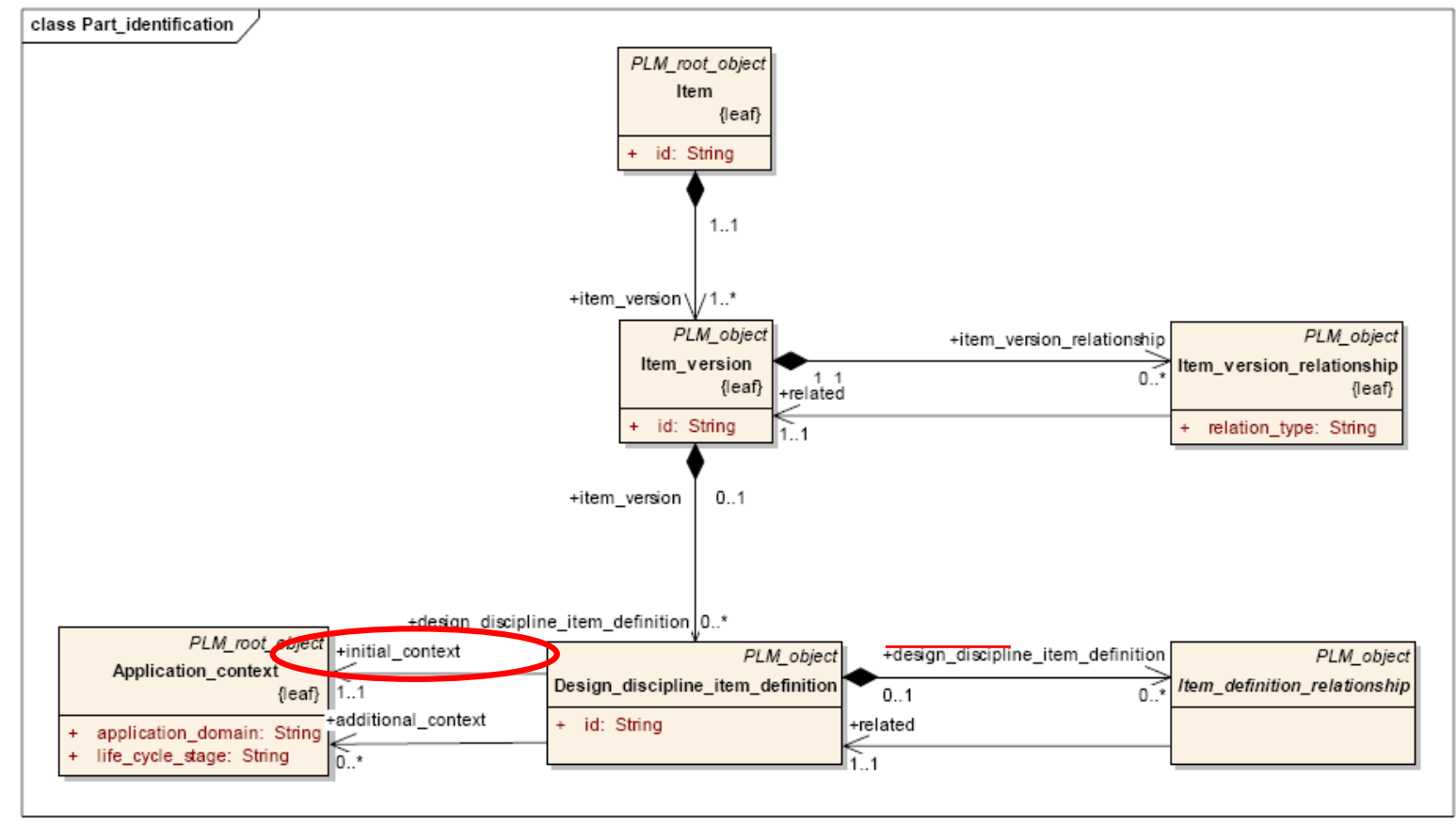

Figure 1: UML version of the informational model in OMG PLM Services: part identification package

In this example, the UML class Design_discipline_item_defintion, representing a view of an item version, contains an association (intitial_context) with the class Application_context that describes the universe of discourse in which the view has been primarily designed. Unfortunately, the equivalent version of Design_discipline_item_defintion in XML contains only an element of 
the type IDREF. The fact that the identification (id) of this type should reference an Application_context is only expressed through an annotation. Therefore, some information is lost.

The computational model of OMG PLM Services is derived from PDTNet and PDM Enablers and is first expressed in UML and then translated to WSDL. The computational model captures the functional aspects of the informational model; it represents the queries that are necessary to navigate it. Examples of these queries are i) the Item_version_query that provides all the versions of a selected item, ii) the Product_detail_query that provides detail information about a product selected through its id and iii) the Item_classification_query that provides the classification of a selected item.

Since the computational model is strictly dependent on the informational model, the project team decided to initially neglect the computational model and to focus only on the informational model. 27 use cases are described in the OMG PLM Services specification to illustrate the context and way of usage of the informational model. These use cases fulfill the requirements analysis reported in the PDTNet project.

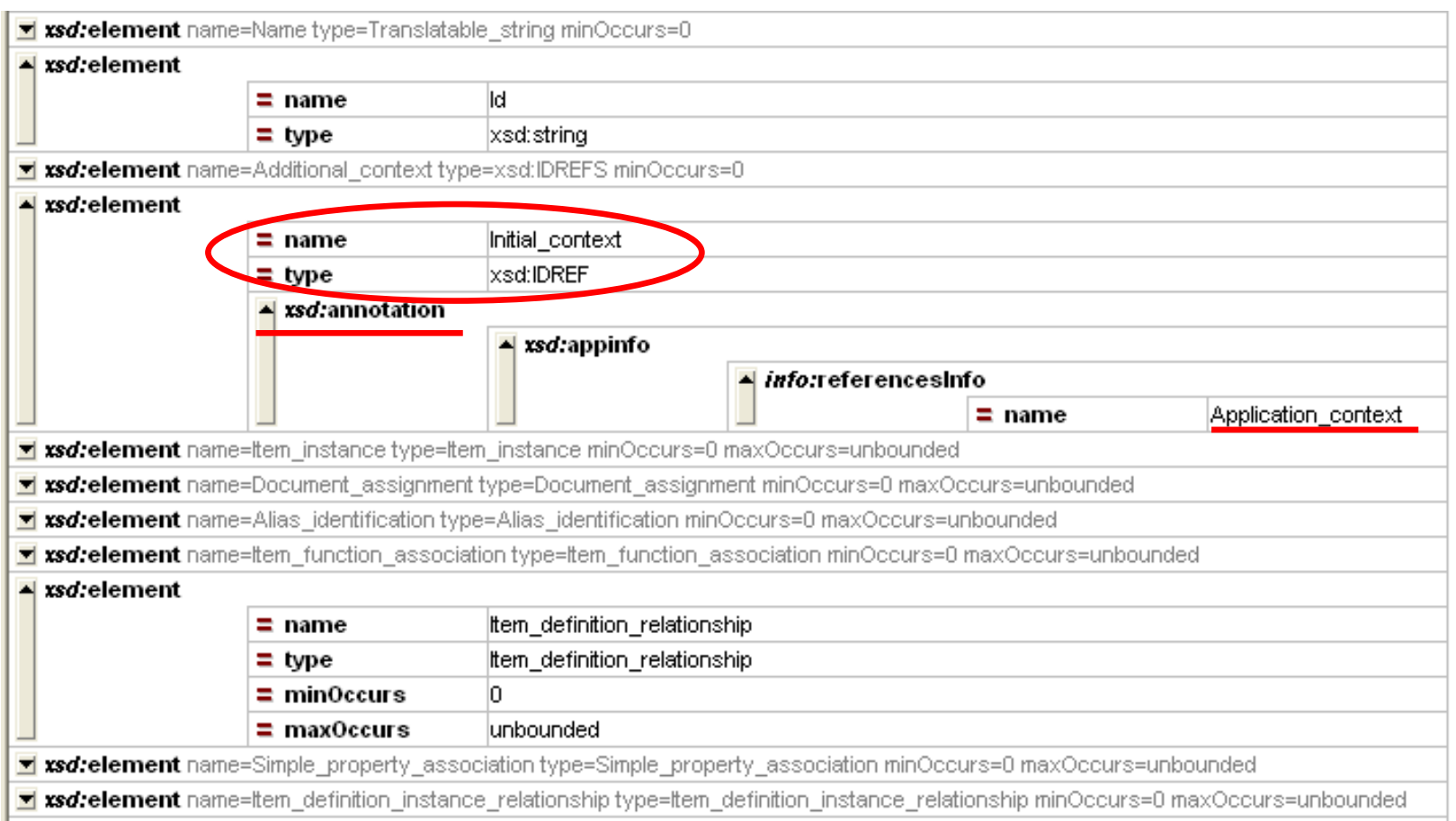

Figure 2: The corresponding XML version

\subsection{OAGIS in brief}

The OAGIS standard aims to achieve interoperability between disparate enterprise-wide business systems by standardizing the architecture of the messages they exchange. These template-based messages, called Business Object Documents (BODs), are defined in XML and exchanged between software applications and/or databases.

BODs are designed as business messages or business documents that are exchanged between software applications or components, between companies, across supply chains, and between supply chains. The contents of a BOD are graphically illustrated in Figure 3. The application 
area contains the information an application needs to communicate the message; it includes information such as the sender characteristics, the creation date of the BOD, and the BOD Globally Unique Identifier. The data area carries the business-specific data communicated by the BOD. The Verb identifies the action being performed on the specific Noun of the BOD. The Noun identifies the business specific data that is being communicated. All of them are defined using XML schema. Each Noun comprises components, which are extensible building blocks and contain compounds and fields. Compounds are basic, shared building blocks that are used by all BODs; these compounds are extensible through contextual use but not with additional fields. Fields are the lowest level elements defined in OAGIS; these fields are the fundamental elements that are used to create the compounds.

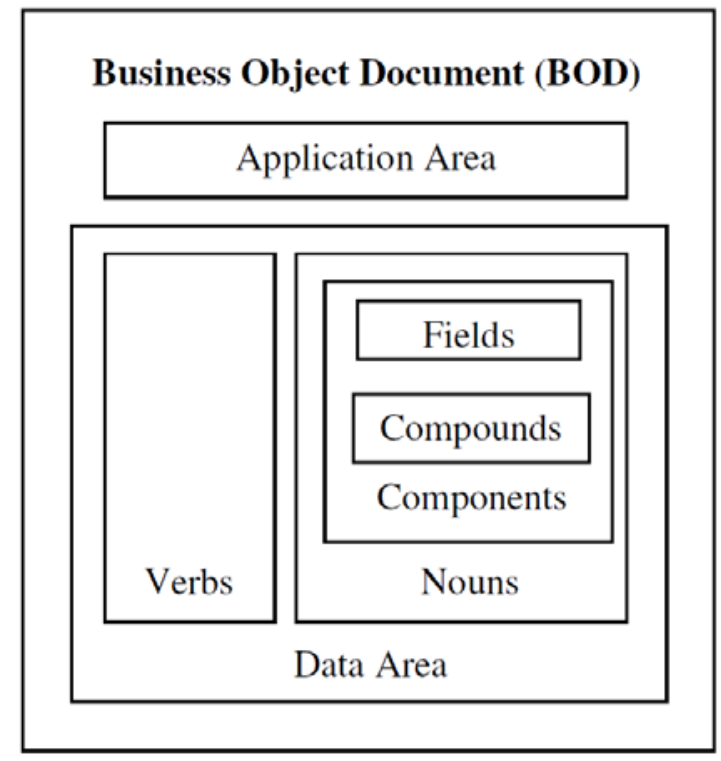

Figure 3: Contents of a Business Object Document (BOD) [3]

The OAGIS Release 9.2 [3] specification contains 13 verbs (e.g., create, get, show, and cancel) and 79 nouns (e.g., BOM, CreditStatus, InventoryBalance, and SalesOrder). While the verb names always represent an action, the nouns names are sometimes a combination of an action and a noun (e.g., PurchaseOrder, InspectDelivery and ReceiveDelivery). The BODs names are then composed by the name of the verb and the name of the noun (e.g., GetBOM, CancelInventoryBalance, ShowPurchaseOrder). The "OAGIS 9 Naming and Design Rules Standard” document [18] conforms to UN/CEFACT [19] XML Naming and Design Rules [20]. It defines the naming, design rules, and guidelines applied by OAGIS to create the XML files representing the BODs. As an example, consider the GetItemMaster BOD, which is of particular interest for our following discussion (Figure 4). 


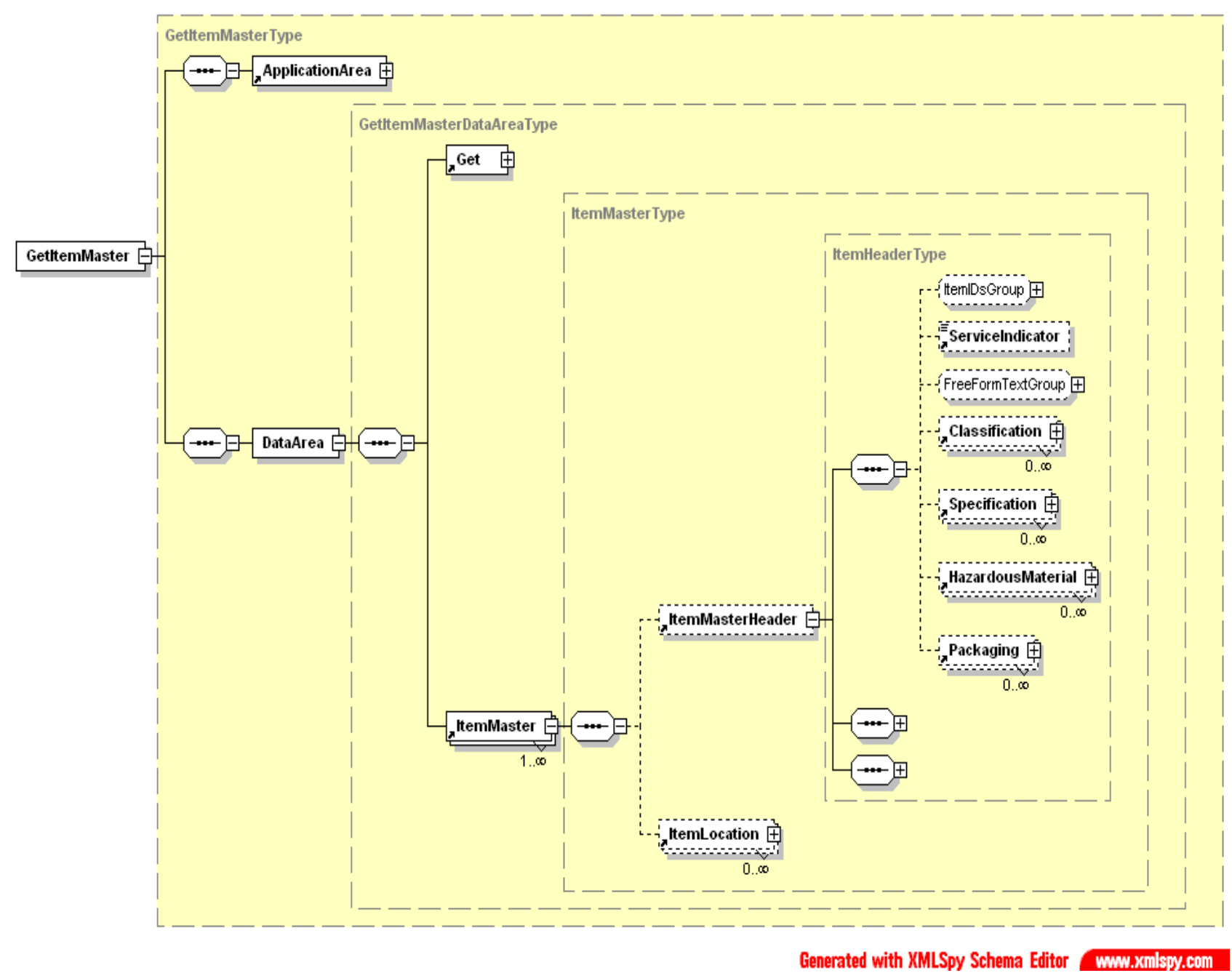

Figure 4: The GetItemMaster BOD

The data area of this BOD contains the verb, Get, and the noun, ItemMaster. The ItemMaster noun represents "any unique purchased part or manufactured product" and includes information such as UPCID code, product classification and packaging type. The Get verb is used when the sender application requests an existing ItemMaster. Usually, a Get-based BOD waits for a Showbased BOD (Show is verb) as a reply. To find the ItemMaster of interest, the message contains a selection criterion that enables searching within all the primary ids of the ItemMasters (belonging to the ItemIDsGroup in the picture).

The OAGIS Release 9.2 specification includes the description of over 200 BODs, and the explanation of 61 integration scenarios, in which two or more integrated applications exchange BODs messages.

\section{Observations and suggestions}

\subsection{Common concepts between OMG PLM Services and OAGIS}

As a starting point for the harmonization of the two standards, the team decided to focus on the mapping between the nouns of the OAGIS BODs and the informational model of OMG PLM 
Services. Unfortunately, even though both the standards have an XML version, their XML schemas and XML schema files (.xsd) are differently organized. While the XML file of the OMG PLM Services informational model looks flat, the XML file of the OAGIS BODs has a complex structure (e.g., it often contains xsd:group). Since changing the XML structure of OMG PLM Services is not the focus of this project, the team decided to match the structure of OAGIS to that of PLM Services.

From the preliminary analysis, it was clear that the two standards are different not only in their organization, but also in their scope and point of view. OAGIS and STEP with its spin-offs (OMG PLM Services included) model different aspects of product data. STEP models mainly focus on Engineering Objects (EOs), which are outputs of design authoring tools (e.g., CAD and PDM) and generally include information such as product geometry and tolerances. OAGIS nouns mainly focus on Business Objects (BOs), which are outputs of business authoring tools and generally include information such as product location, cost, and sales order. Some examples of business authoring tools include ERP and Supply Chain Management (SCM).

Since EOs and BOs usually complement each other, the challenge to the team was to find the product data that travel across engineering authoring tools and business authoring tools. The team found that the intersection between the engineering and the business domains is constituted by the following OAGIS nouns: EngineeringChangeOrder, BOM, and ItemMaster.

The EngineeringChangeOrder noun represents the document used to request a change to a manufactured product. The requested change pertains to the engineering aspects of the product, i.e., its structure and shape, and when it is first suggested, then reviewed and finally approved. The EngineeringChangeOrder noun contains information on both the metadata of the document, such as document ID and creation date, and the content of the document itself, such as the estimated engineering cost of the change and the suggested new BOM of the product to be changed.

The BOM noun represents the document describing the structure of a product. Again, this noun contains information of both the metadata of the document, such as its creation date, and its content, such as the components of the product. The document mainly describes the engineering and manufacturing Bill Of Material of the product.

As said earlier, the ItemMaster noun represents any unique purchased part or manufactured product. The product information represented by this noun is generated and processed by applications belonging to different domains: engineering, manufacturing, logistics, customer relationships, financials, etc. Table 1 contains, for each application domain, a few examples of the product information represented in the ItemMaster noun; a description of this information is also provided.

Table 1: Examples of the product information represented in the ItemMaster noun

\begin{tabular}{|l|l|l|}
\hline \multirow{2}{*}{ Application domain } & $\begin{array}{c}\text { Product information represented } \\
\text { in the ItemMaster noun }\end{array}$ & \multicolumn{1}{|c|}{ Description } \\
\hline \multirow{4}{*}{ Engineering } & Specification & Properties or features of the product \\
\cline { 2 - 3 } & DrawingAttachment & Document that graphically represents the product \\
\cline { 2 - 3 } & BOMReference & Product structure \\
\hline \multirow{3}{*}{ Manufacturing planning } & Routing & Receipt routing of the product \\
\cline { 2 - 3 } & AvarageRunSizeQuantity & Average quantity made during a manufacturing run \\
\cline { 2 - 3 } & LeadTimeDuration & Forecasted time used to calculate the product availability \\
\hline
\end{tabular}




\begin{tabular}{|l|l|l|}
\hline \multirow{4}{*}{ Application domain } & $\begin{array}{c}\text { Product information represented } \\
\text { in the ItemMaster noun }\end{array}$ & Description \\
\hline \multirow{4}{*}{ Manufacturing execution } & ExecutionTimePeriod & Time period in which the product is effectively in process \\
\cline { 2 - 3 } & EffectiveTimePeriod & Time period in which the Item is effective \\
\cline { 2 - 3 } Logistics & ManufacturerItemID & Identifier of the product provided by the manufacturer \\
\hline \multirow{4}{*}{ Sales } & FreightClassification & Product classification based upon its transportation way \\
\cline { 2 - 3 } & Packaging & Packaging information including type and dimension \\
\cline { 2 - 3 } & StorageUOMcode & Storage unit of measure of the product \\
\hline \multirow{5}{*}{ Environmental information } & UPCID & Barcode of the product used in stores \\
\cline { 2 - 3 } & CustomerItemId & Identifier of the product provided by the customer \\
\cline { 2 - 3 } & HazardousMaterial & Hazardous material code and description \\
\cline { 2 - 3 } & OwnerParty & Activated when the product needs to be tracked \\
\hline \multirow{3}{*}{ Financial } & UnitValue & Who owns and is responsible for the product \\
\cline { 2 - 3 } & GLEntityID & The monetary value of the product \\
\cline { 2 - 3 } & GLAccount & General ledger account \\
\hline
\end{tabular}

The purpose of this table is neither to exhaustively list all the product information represented in ItemMaster, nor to cover all the application domains. The purpose of this table is to show the reader the variety of information included in this noun.

\subsection{Modeling paradigm in OMG PLM Services and OAGIS}

The information concerning the engineering domain is of a particular interest for this project; it represents the intersection between the ItemMaster noun and elements in the OMG PLM Services informational model. This intersection consists of product engineering aspects that belong to two different levels of abstraction: real world products and product models (see Figure 5). 


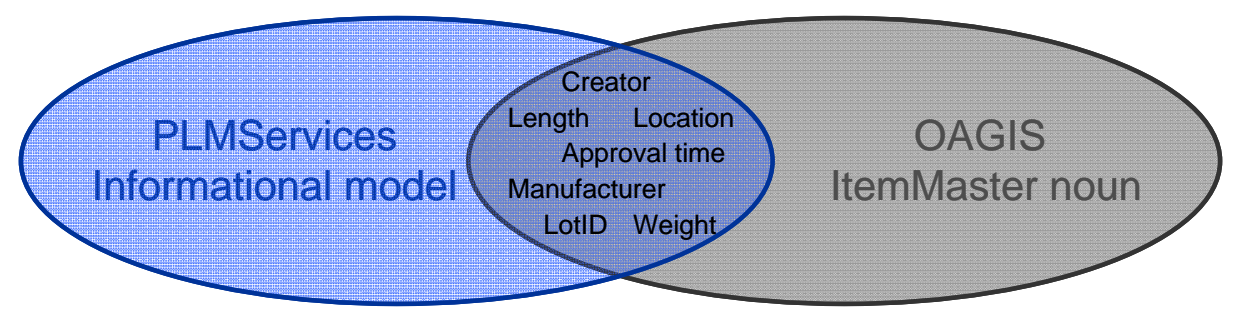

\section{INSTANTIATION}

Product model: mouse model

Real world product: my_mouse
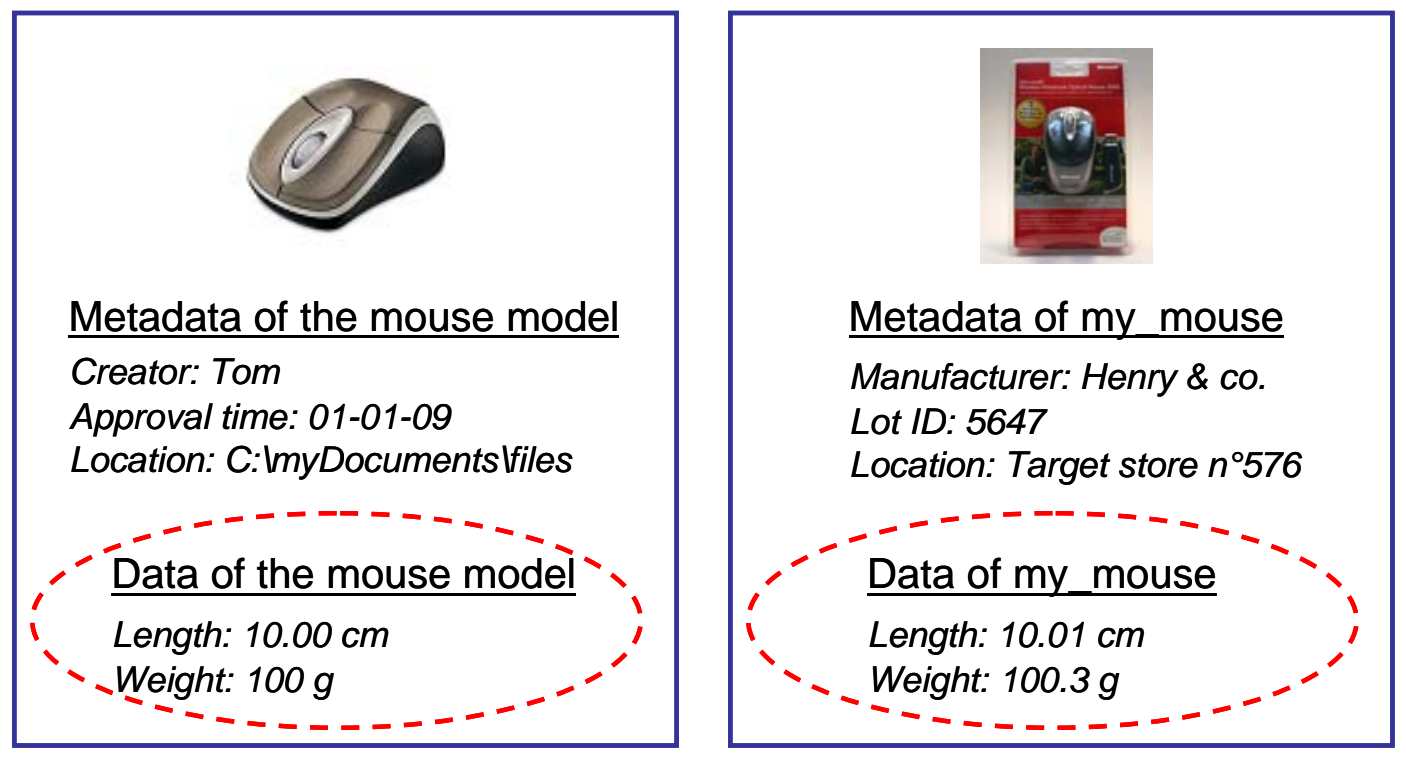

Figure 5: Real world products and product models data

Real world products are objects that can be seen and touched and exist in the real world: my mouse in Figure 5 is a real world object. Standards that represent real world products need to include both the metadata of the real objects, such as manufacturer and location, and the data such as length and weight. The data value of real world products $(10.01 \mathrm{~cm}$ and $100.3 \mathrm{~g}$ in the figure) will have a direct correspondence with the data value represented in the product models (10.00 cm and $100 \mathrm{~g}$ in the figure). The metadata of the mouse model represent information such as the name of the model creator and the location of the model file.

In OAGIS, the ItemMaster noun mainly represents a real world product ("a unique purchased part or manufactured product") but also includes information of the product model: the metadata and data of both the real world product and its model are flattened in a unique noun.

In OMG PLM Services, the informational model mainly represents product models: its instantiation is often done by CAD applications. Figure 6 represents, in UML, how the product model is connected to the real world products. 


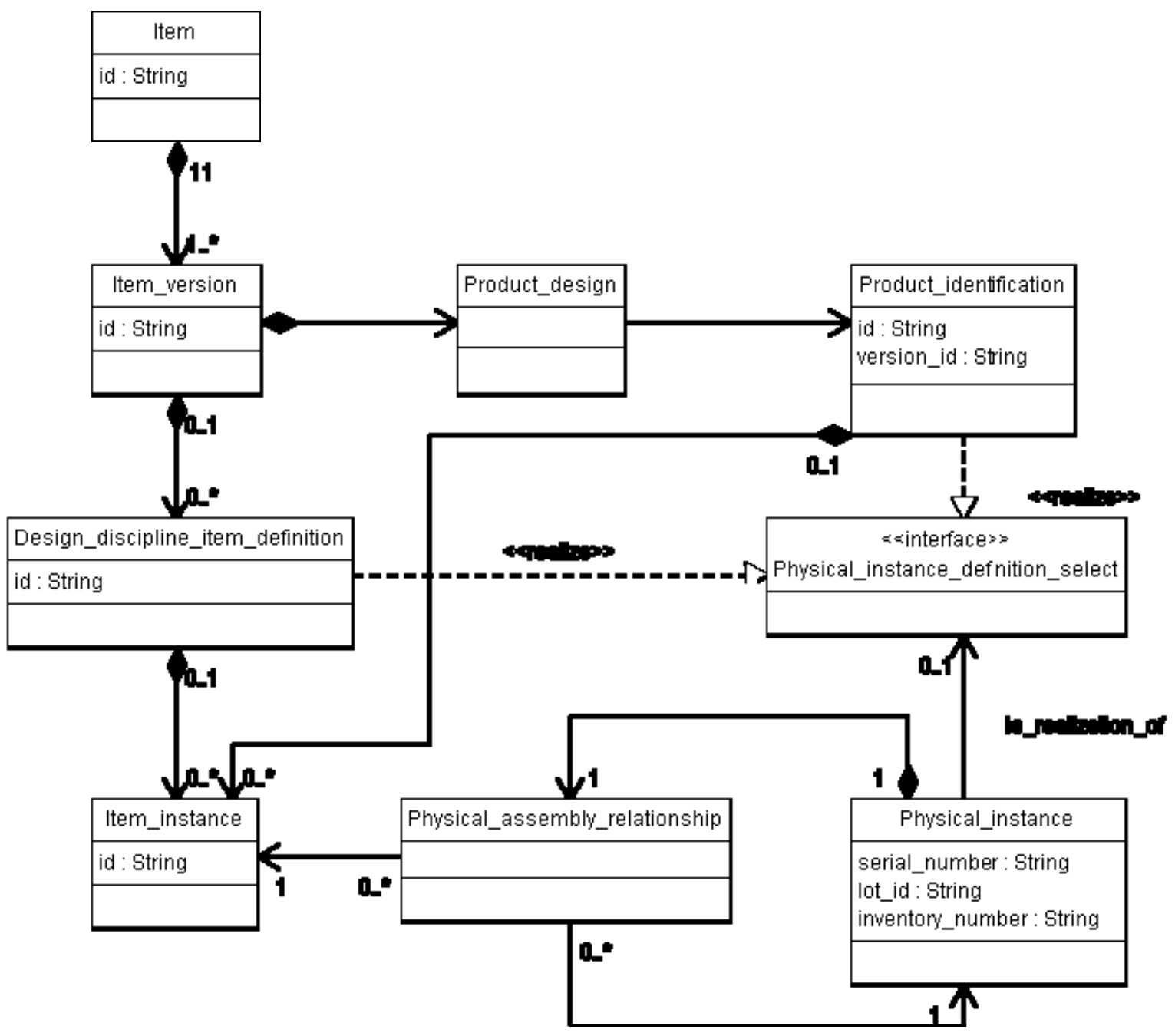

Figure 6: Product model and real world product in OMG PLM Services

The class Item represents the product model information that is common among all the item versions. Item_version, the class representing the product versions, collects all the information characterizing a physically realizable object: in practice it represents the product model of each version of a product. Each product version is depicted through several views; examples of these views are the assembly definition, collection definition or mating definition. The Design_discipline_item_definition class supports the representation of these views. Each view contains one or more Item_instances: they represent the occurrences of a product in a bill of material. An Item_instance is defined either by a Design_discipline_item_definition or by a Product_identification. A Product_identification identifies a manufacturable object and is connected to the product version through the class Product_design.

The occurrence of a product in a bill of material can have its correspondent in the real world: the class Physical_instance represents a real world product. This real world product is connected through the class Physical_assembly_relationship to its super-assembly, which itself will be a Physical_instance. Physical_assembly_relationship is then connected to the Item_instance that 
the physical component is the realization of. Each Physical_instance is finally defined by a product model: the classes Product_identification or Design_discipline_item_definition collect the information defining the Physical_instance (Physical_instance_definition_select interface).

The class Physical_instance represents the core concept for realizing the mapping between OAGIS and OMG PLM Services. Physical_instance corresponds to the engineering information contained in the ItemMaster noun and related to the real world product.

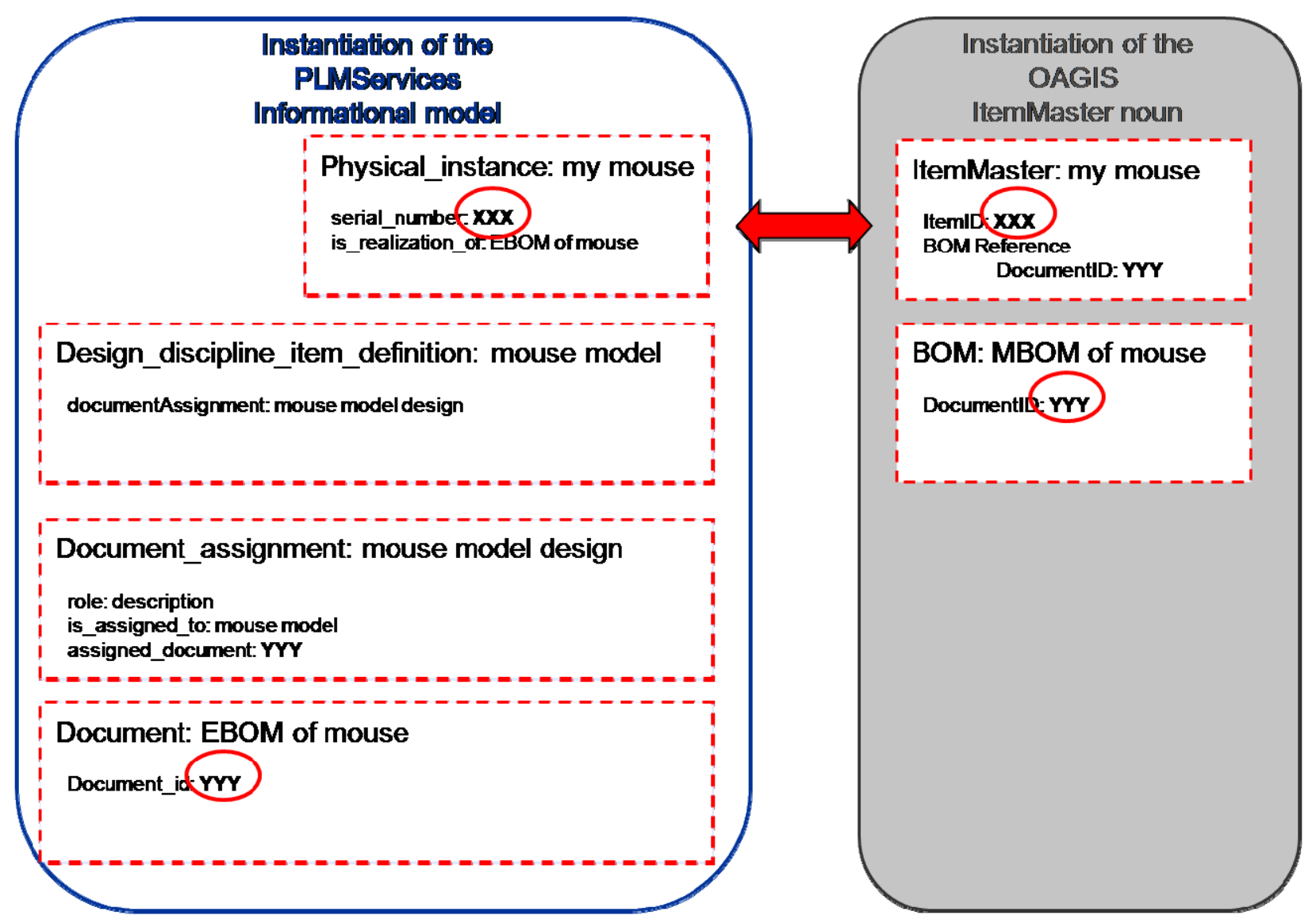

Figure 7: Physical_instance as a core concept for realizing the mapping

In Figure 7 the red arrow represents the first possible mapping between OMG PLM Services and OAGIS. The team implemented this mapping, as a proof of concept, by using XSLT (EXtensible Stylesheet Language Transformations) [21] files generated through MapForce ${ }^{\mathrm{TM}}$ [22]. An alternative mapping method, finally rejected by the team, used an Excel file to organize the XPath [23] expressions indicating the same concepts in the two standards.

Figure 8 is a screenshot of MapForce: the two boxes at the center of the figure show in a tree representation the XMLschema files of the standards and the lines connecting them represent the mapping between the elements in each tree. The ItemMaster noun is mapped into the Physical_instance class and the primary ID of the ItemMaster is mapped into the serial_number attribute of Physical_instance. The serial_number attribute is defined by OMG PLM Services as the identifier that distinguishes one Physical_instance from another. 


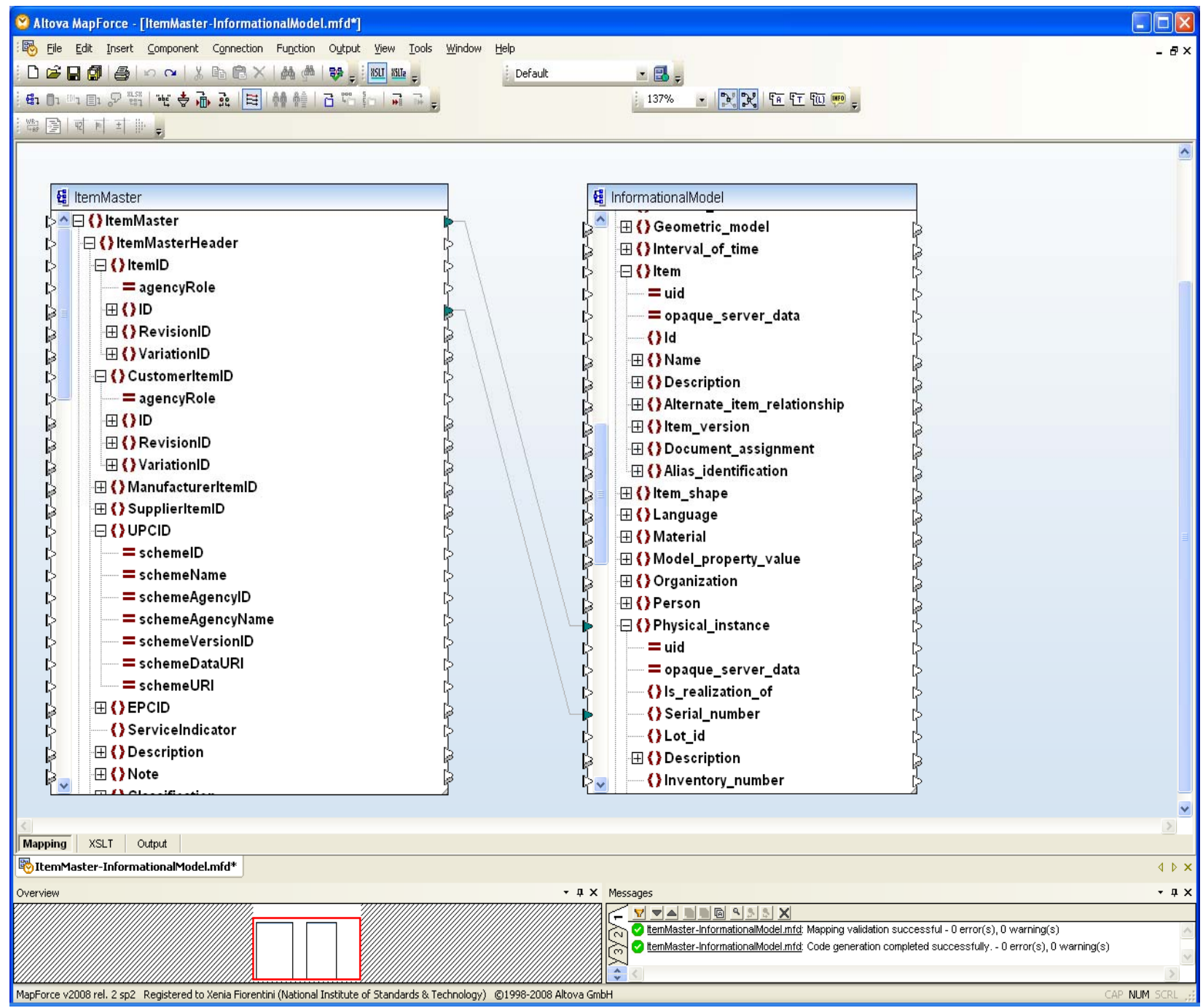

Figure 8: MapForce screenshot of the Physical_instance mapping

This first mapping provides the opportunity of testing the chosen mapping method and proves the feasibility of the goals set for this project. Since in OMG PLM Services there are few classes dedicated to the representation of real world products, this mapping covers, only to a limited extent, the intersection between OMG PLM Services and OAGIS.

The major part of this intersection is related to the representation of product models, depicted in Figure 6 as EBOM for OMG PLM Services and MBOM for OAGIS. The project challenge is then to identify which information is common between the representation of product models in OMG PLM Services and OAGIS.

\subsection{Scenarios}

To address this issue the team decided to build a scenario ${ }^{1}$ in which product models are instantiated and exchanged by two applications using the two standards. Both the standards contain, as part of their specification, the description of several scenarios, some of which already

\footnotetext{
${ }^{1}$ For the purpose of this report, the terms scenario and use case are interchangeable.
} 
include the engineering change management. We report in Table 2 some examples of the scenarios contained in the specifications.

Table 2: Examples of use cases in OMG PLM Services and scenarios in OAGIS

\begin{tabular}{|c|c|}
\hline Use cases in OMG PLM Services & Scenarios in OAGIS \\
\hline $\begin{array}{l}\text { - } \text { Export/import of assembly data } \\
\text { - } \text { Browsing up/down the product structure } \\
\text { data } \\
\text { - } \quad \text { Browsing of alternative solutions } \\
\text { - } \quad \text { ECMange notification } \\
\text { ECM participants comments }\end{array}$ & $\begin{array}{l}\text { - } \text { Catalogue exchange } \\
\text { - } \quad \text { ERP to finite scheduling and manufacturing } \\
\text { execution } \\
\text { - } \quad \text { Plant data collection } \\
\text { - } \quad \text { Production synchronization } \\
\text { Engineering changes }\end{array}$ \\
\hline
\end{tabular}

A comparison between these scenarios is hardly achievable since they strongly differ in scope and granularity. While the OMG PLM Services use cases model the engineering aspects of product-related data, the OAGIS scenarios model the business aspects. Moreover, the data needed to realize the OMG PLM Services use cases have a fine granularity, while the data needed for the OAGIS scenarios have a high level of aggregation.

The most suitable scenario for this challenge is constituted by the Engineering Change Management (ECM). The communication, proposal, and acceptance of such changes represent the bridge between the manufacturing and the engineering worlds. In this scenario, during the manufacturing stage of products, some alterations to the product model need to be made. These alterations will affect the representation of the product model in both the engineering authoring tools (using OMG PLM Services) and the business authoring tools (using OAGIS).

The OMG PLM Services specification includes four use cases for ECM: ECM participant proposal for a change, ECM participant comments, ECM participant approval and ECM participant detailing and comments. These uses cases were defined according to the Recommendation for Engineering Change Management Part 1: Engineering Change request (ECR) proposed by VDA [24], the German automotive industry association. Therefore these use cases do not include Engineering Change Order (ECO). These use cases provide a list of the messages exchanged by the actors for ECR; [24] describes how these messages are related to the informational model of OMG PLMServices.

The OAGIS specification includes one scenario dedicated to ECM: scenario 49 "Engineering changes” (see Figure 9). 


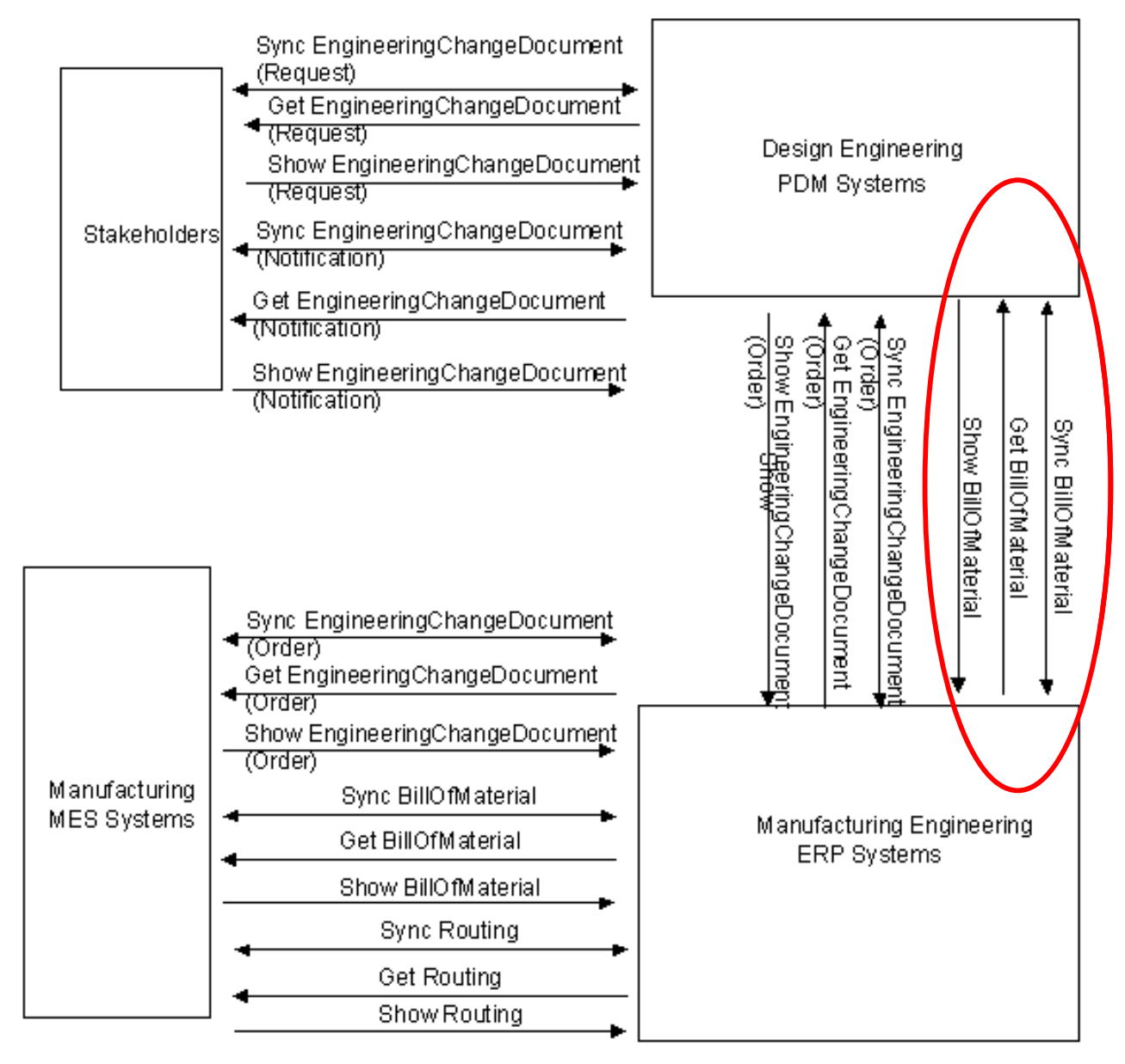

Figure 9: ECM scenario in OAGIS

In this scenario one or more design engineering systems are integrated with other manufacturing engineering systems and exchange BODs relative to the following nouns: EngineeringChangeOrder, BOM, and Routing. The engineering change request is originated by any department (stakeholders) and transferred to the engineering department. Once the engineering department approves the request, a notification of approval is sent from the PDM system to the stakeholders and the order for the execution of the change is sent to the ERP system, along with the new BOM of the product. The engineering change order is finally communicated to the manufacturing execution systems.

Scenario 49 seems to address the requirements for the integration scenario of this project as it is focused on engineering information and it describes the integration of engineering and manufacturing systems, i.e., PDM and ERP systems, respectively. For the sake of this project, the team restricted this scenario to the exchange of the BOM messages between design engineering systems and manufacturing engineering systems (red oval in Figure 9).

In this restricted scenario, a PDM system conforming to the OMG PLM Services standard exchanges the BOM information with an ERP system. While the PDM system contains the product EBOM, the ERP system needs the product MBOM to generate the manufacturing planning and the purchase orders. Figure 10 describes this scenario. 


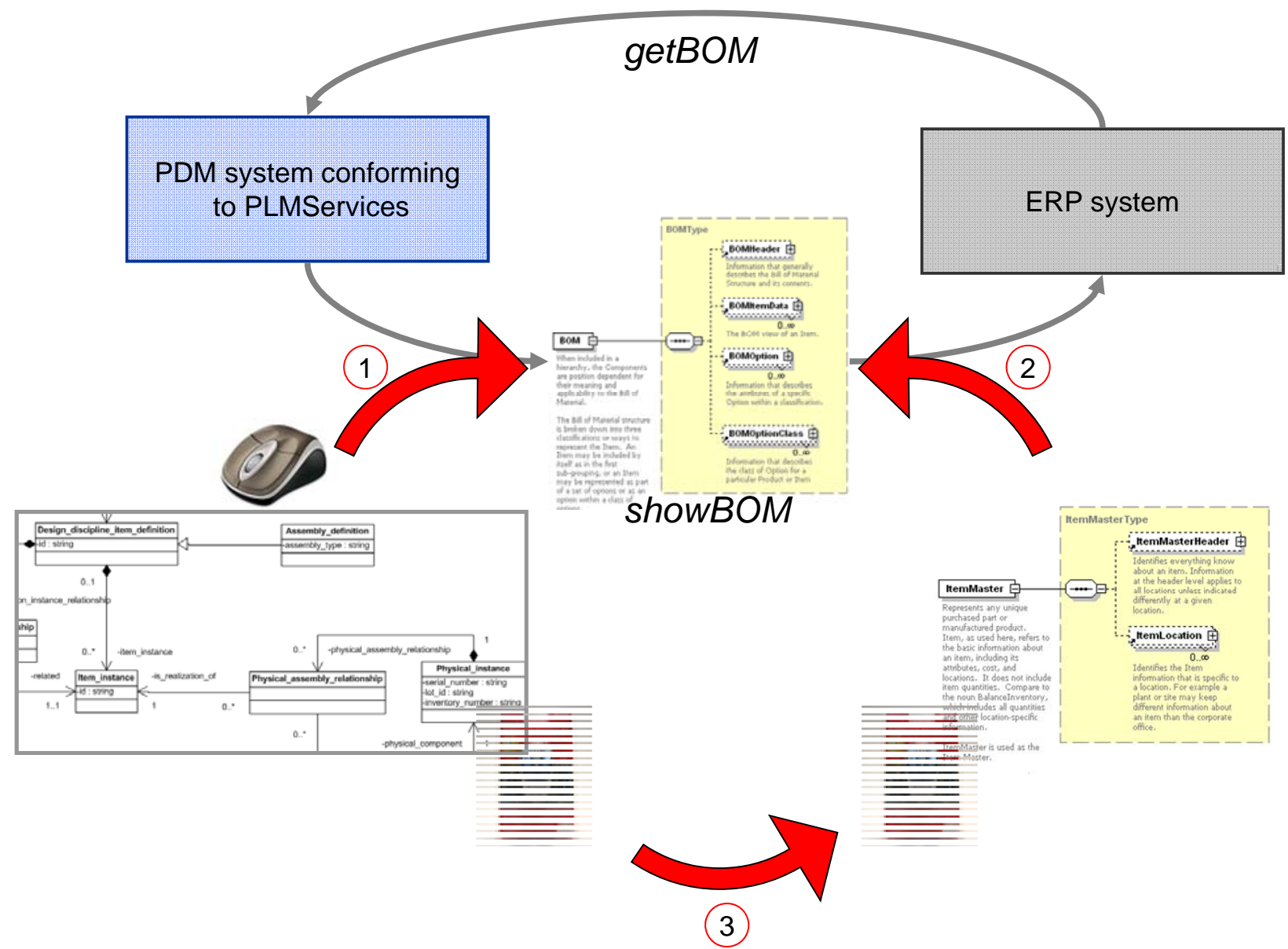

Figure 10: A scenario for the harmonization

The ERP system requires a BOM from the PDM system (getBOM BOD): the PDM system replies to this request by showing the BOM (showBOM BOD). To realize this scenario, the data of the PDM system, conforming to OMG PLM Services, need to be aggregated into the BOM noun. The research objective is then to understand how the information from OMG PLM Services is aggregated into the BOM noun.

The mechanism of aggregation of the BOM noun is shown in red arrows in Figure 10:

1) Some information in the BOM noun can be directly aggregated from the OMG PLM Services output file. For example, the Child/ParentBOM in the BOM noun could correspond to the product structure in OMG PLM Services (package Part Structure)

2) Some information in the BOM noun is shared with the ItemMaster noun. For example, the information contained in ItemIDsGroup, Classification, Specification, and BOMReference are in common between the BOM and ItemMaster nouns.

3) Some information in the ItemMaster noun can be aggregated from the OMG PLM Services file. For example, as previously explained, serial_number of Physical Instance in OMG PLM Services have a direct correspondent in ItemMaster.

Understanding these three mechanisms becomes then the part of the future work of the team. 


\section{Issues and Future Plans}

The initial work presented in this report contributed to reaching the following objectives:

- Understanding each standard by identifying its scope, organization, and modeling philosophy

- Designing one or more use cases (scenarios) to understand the integration issues

- Recognizing the concepts that intersect both the standards

- Identifying portions of the models that are part of the intersection

- Realizing the harmonization

In summary, the first part of the work was dedicated to prove the feasibility of harmonization, to define the research directions for its implementation and to understand the issues connected to the long-term objectives of the project, which is to develop a method for comparing and harmonizing standards.

The main issue is about the different approaches adopted by the two standards towards modeling product information. The approach of OMG PLM Services is top-down: the informational model first defines a product model and then links it to actual products (or real world products). On the other hand, the approach of OAGIS is bottom-up: the information contained in the selected nouns mainly represents actual products and contains references to product models.

These different approaches arise from the different domains addressed by the two standards. OMG PLM Services was conceived to be used in the engineering domain, where product models are planned and designed. As a consequence, the engineering objects described in OMG PLM Services mainly represent product models. OAGIS has a different prospective, covering other domains such as manufacturing, logistics and sales, and the information of real world products is the main focus. OAGIS flattens the real world object information and product model information as a unique model.

The challenge still remains as to how to connect the representations of product models and actual products in a structured and formal fashion so as to accomplish the harmonization. To address this challenge we could explore the ideas implemented in the Athena project [25]. In the Athena project, the harmonization between proprietary formats and the standard BODs within the inventory domain is realized by creating an e-Kanban reference ontology [26]. The proprietary formats and the standard BODs are then mapped to this ontology, as a way to achieve harmonization.

The team will also explore the possibility of creating a BOM reference ontology, to which both the PLM Services schema and BODs schema could be mapped. The BOM reference ontology should represent both the engineering and manufacturing BOM, and should incorporate information about both product models and real world products at different levels of granularity. This requires a clear and formal model of the BOM. This ontology could be organized in a multilayered architecture similar to MOF (Meta Object Facility) [27]. The concepts in each layer and the relationships between layers could be semantically defined. Semantic technologies could enable the harmonization; for example, languages based on description logic could enhance the formalization and allow the consistency checking between schemas and instances [28]. Once the 
PLM Services and BODs schemas are mapped to the appropriate layer of the BOM reference ontology, the direct mapping between PLM Services and OAGIS can be accomplished.

To realize this, the team would need, as a first step, to analyze the concepts and requirements for the BOM reference ontology, paying particular attention to the representation of product models, actual products, and their relationships. For this analysis, the team could consider the scenario presented in the previous section: the BOM reference ontology would represent all the concepts necessary for the realization of that scenario.

As a second step, the team would need to select a language expressive enough to represent those concepts. OWL-DL [29] or RDF [30] could be two of the candidate languages. To realize the mapping, these languages should be used not only to represent the BOM reference ontology, but also the PLM Services and BODs schemas.

A first attempt towards a semantic version of STEP has already been presented in the OntoSTEP project [31]. In this project, the STEP EXPRESS schemas and their instantiations are translated into OWL-DL. In the future, the team could explore the opportunity of creating a semantic version of the OMG PLM Services informational model by applying the same principles adopted for the OntoSTEP project. In a similar way, an RDF version of some OAGIS BODs has already been created for the Athena project. The tool used in this project, i.e., AXTOR [25], could be exploited to generate the RDF versions of the ItemMaster, BOM, and EngineeringChange nouns. The OntoSTEP and Athena projects could then represent the starting point for “ontologizing” the PLM Services and OAGIS standards, respectively.

As a third step, the "ontologized" versions of PLM Services and OAGIS should be mapped to the BOM reference ontology. First, for the purpose of harmonization, the team will identify the concepts in the BOM reference ontology that are part of the intersection between the two standards. Second, to realize the scenario, the team will identify the ownership of the concepts that are not part of the intersection but are still represented in the BOM reference ontology. For example, the data related to the supplier identification and lot size are represented in the BOM and ItemMaster nouns but do not have an equivalent in PLM Services; these data will be then owned by OAGIS. As another example, the data related to tolerances and assembly relationships are represented in PLM Services but do not have an equivalent in OAGIS; these data will then be owned by PLMServices.

The principles and ideas applied in this project, its results and the experience gained during its realization could finally be summarized to achieve long term goals: the team could develop a generic methodology for comparing and harmonizing information standards. This methodology will be based on semantic technologies and will be aligned with the vision of both the standards and semantics communities [32].

Disclaimer: No approval or endorsement of any commercial product by NIST is intended or implied. Certain commercial equipment, software, instruments or materials are identified in this report to facilitate better understanding. Such identification does not imply recommendations or endorsement by NIST nor does it imply the materials, software, or equipment identified are necessarily the best available for the purpose.

Acknowledgement: The authors would like to acknowledge the comments and suggestions of Professor Steven Fenves, NIST Associate, in improving the report but any residual mistakes remain ours. 


\section{References}

[1] ISO 10303-1:1994, Industrial automation systems and integration -- Product data representation and exchange -- Part 1: Overview and fundamental principles.

[2] OMG PLM Services v2.0. http://www.omg.org/cgi-bin/doc?dtc/07-05-01, 2007

[3] Open Applications Group Integration Specification. www.oagi.org, 2008

[4] The Object Management Group (OMG). http://www.omg.org/, 2009

[5] OASIS: Organization for the Advancement of Structured Information Standards. www.oasisopen.org, 2006

[6] The Open Application Group (OAGi). www.oagi.org, 2009

[7] Srinivasan, V., "An Integration Framework for Product Lifecycle Management," ComputerAided Design, Vol. In Press, 2009.

[8] Model-Driven Architecture. http://www.omg.org/mda/, 2004

[9] ISO 10303-214:2003, Industrial automation systems and integration -- Product data representation and exchange -- Part 214: Application Protocol: Core data for automotive mechanical design process.

[10] PDTNet, Project Information. http://www.prostep.org/en/standard-info/pdt-netprojekt.html, 2000

[11] PDM Enablers v1.3, Final Report. http://www.omg.org/cgi-bin/doc?dtc/2000-06-02, 2000

[12] eXtensible Markup Language (XML). http://www.w3.org/XML/, 2005

[13] Web Services Description Language (WSDL) 1.1. http://www.w3.org/TR/wsdl, 2001

[14] ISO 10303-11:2004, Industrial automation systems and integration -- Product data representation and exchange -- Part 11: Description methods: The EXPRESS language reference manual.

[15] ISO 10303-14:2005, Industrial automation systems and integration -- Product data representation and exchange -- Part 14: Description methods: The EXPRESS-X language reference manual.

[16] XMI Mapping http://www.omg.org/technology/documents/formal/xmi.htm, 2007

Specification. 
[17] UML 2.0 Superstructure Specification. http://www.omg.org/cgi-bin/doc?ptc/03-08-02, 2003

[18] Minakawa, G., Ramanathan, S., and Rowell, M.. OAGIS 9 Naming and Design Rules Standard. Open Applications Group, 2005.

[19] The United Nation Centre for Trade Facilitation and Electronic Business (UN/CEFACT). http://www.unece.org/cefact/, 2009

[20] M.Rowell.. UN/CEFACT XML Naming and Design Rules Version 3.0. United Nations Centre for Trade Facilitation and Electronic Business, 2008.

[21] XSL Transformations (XSLT) Specification. http://www.w3.org/TR/xslt, 1999

[22] MapForce. http://www.altova.com/products/mapforce/data_mapping.html, 2009

[23] XML Path Language Specification. http://www.w3.org/TR/xpath, 1999

[24] Recommendation for Engineering Change Management Part 1: Engineering Change request (ECR) http://www.vda.de/en/publikationen/publikationen_downloads/detail.php?id=382\&PHPSESS

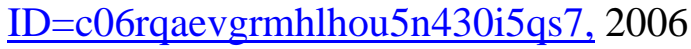

[25] ATHENA project: Advanced Technologies for interoperability of Heterogeneous Enterprise Networks and their Applications. http://www.athena-ip.org/, 2004

[26] Barkmeyer, E. J., and Kulvatunyou, B., "An Ontology for the e-Kanban Business Process," National Institute of Standards and Technology, NISTIR 7404, 2007.

[27] Meta Object Facility (MOF) Specification. http://www.omg.org/docs/formal/02-0403.pdf, 2002

[28] Fiorentini, X., Rachuri, S., Mani, M., Fenves, S. J., and Sriram, R. D., "An Evaluation of Description Logic for the Development of Product Models," National Institute of Standards and Technology, NISTIR 7481, 2008.

[29] Web Ontology Language (OWL). http://www.w3.org/2004/OWL/, 2005

[30] Resource Description Framework (RDF). www.w3.org/RDF/, 2006

[31] Krima, S., Barbau, R., Fiorentini, X., Rachuri, S., and Sriram, R. D., "OntoSTEP: OWLDL Ontology for STEP," National Institute of Standards and Technology, NISTIR 7561, 2009.

[32] OntologySummit 2009 Communiqué: Toward Ontology-based Standards. http://ontolog.cim3.net/cgi-bin/wiki.pl?OntologySummit2009_Communique, 2009. 